\title{
Rectal Stenosis, CTCAE
}

National Cancer Institute

\section{Source}

National Cancer Institute. Rectal Stenosis, CT CAE. NCI Thesaurus. Code C143803.

A disorder characterized by a narrowing of the lumen of the rectum. 